\title{
Verzeichniss der Mitglieder
}

\section{der \\ pädiatrischen Section.}

Abelin, Dr. Prof. Stockholm. Baginsky, A., Dr. Berlin. Barwinski, Dr. Suderode. Beneke, Dr. Geh. Med. - R. Prof. Marburg.

Binz, Dr. Prof. Bonn.

$B o h n$, Dr. Prof. Königsberg.

Bokai, Dr. Prof. Pest.

Demme, Dr. Prof. Bern.

Dohrn, Dr. Prof. Marburg.

Edel, Dr. Charlottenburg.

Emminghaus, Dr. Prof. Dorpat.

D'Espine, Dr. Prof. Genf.

Flesch, Dr. San.-R. Frankfurt a. M.

Förster, Dr. Med.-R. Direktor des Krankenhauses. Dresden.

Gerhardt, Dr. Geh. Hofrath. Prof. Direktor der medicinischen Klinik. Würzburg.

Hauke, Dr. dirigirend. Primararzt d. Kronprinz Rudolf Kinderhospitales. Wien.

Hennig, Dr. Prof. Leipzig.

Herz, Dr. Wien.

Koch, Dr. Wiesbaden.

Kohts, Dr. Prof. Strassburg i. E. Kormann, Dr. Coburg.

Leichtenstern, Dr. Prof. Tübingen.

Lichtheim, Dr. Prof. Bern.
Lorey, Dr. Frankfurt a. M.

Löwe, $W$., Dr. Berlin.

Mannkopff, Dr. Prof. Marburg.

Marcus, Dr. Frankfurt a. M.

Monti, Dr. Docent. Wien.

Müller, Dr. Prof. Bern.

Nötzel, Dr. Ob.-Stabs-Arzt. Colberg.

Pauly, Dr. Posen.

Penzoldt, Dr. Erlangen.

Pfeiffer, Dr. Med.-R. Weimar.

Pilz, Dr. Stettin.

Politzer, Dr. Prof. Wien.

Ranke, Dr. Prof. München.

Rauchfuss, Dr. Prof. St. Petersburg.

Rehn, Dr. Frankfurt a. M.

v. Rinecker, Dr. Geh. Hofrath. Prof. Würzburg.

Ritter von Rittershain, Dr. Prof. Prag. Salomon, Max, Dr. Berlin.

Schnitzler, Dr. Prof. Redakteur der Wiener medicinisch. Presse. Wien. Schulze, Dr. Geh. Hof-R. Prof. Jena. Seeligmüller, Dr. Docent. Halle.

Seidel, Dr. Prof. Jena.

Soltmann, Dr. Docent. Breslau.

Stage, Dr. Kopenhagen.

Thomas, Dr. Prof. Freiburg i./Br.

Trüstedt, Dr. Bad Elmen.

Tschamer, Dr. Docent. Graz. 
VIII

Unterholaner, Dr. dirigirend. Primararzt des Leopoldstâdter Kinderspitales. Wien.

Vogel, Dr. Prof. Dorpat.

Vollmer, Dr. San.-R. dirigirend. Arzt des Hedwig-Krankenbanses. Berlin.

Wagner, Dr. Redakteur des Jahrbuches $f$. Kinderheilkunde. Leipzig.
Wertheimber, Dr. Mũnchen. Wiederhofer, Dr. Prof. Wien.

Wiesner, Dr. Frankfurt a. M.

Winckel, Dr. Geh. Hofrath. Prof.

Direktor der Königl. Entbindungs-

Anstalt. Dresden.

Wyss, Dr. Prof. Zürich.

Zini, Dr. Graz.

Der Vorstand der Section pro 1880 besteht aus den Herren

C. Gerhardt (Würzburg).

F. Winckel (Dresden).

W. Löwe (Berlin).

Max Salomon

A. Baginsky

Schriftfübrer. 